\title{
Tanshinone IIA ameliorates diabetic cardiomyopathy by inhibiting Grp78 and CHOP expression in STZ-induced diabetes rats
}

\author{
SHULIANG TAO $^{1}$, LIUYIN CHEN ${ }^{2}$, JINGMEI SONG ${ }^{1}$, NINGNING ZHU $^{3}$, \\ XUEYI SONG ${ }^{4}$, RUOLI SHI ${ }^{5}$, GANGFENG GE ${ }^{1}$ and YUEMING ZHANG ${ }^{1}$ \\ ${ }^{1}$ Basic Medical College $;{ }^{2}$ Fourth Clinical Medical College; ${ }^{3}$ First Clinical Medical College and \\ ${ }^{4}$ Second Clinical Medical College, Zhejiang Chinese Medical University, Hangzhou, Zhejiang 310053; \\ ${ }^{5}$ Community Health Service Center of Sijiqing Street, Hangzhou, Zhejiang 310016, P.R. China
}

Received March 2, 2018; Accepted June 29, 2018

DOI: $10.3892 / \mathrm{etm} .2019 .7580$

\begin{abstract}
Diabetic cardiomyopathy (DCM), one of the common diabetic complications, causes a high rate of mortality in patients with diabetes. Tanshinone IIA (TSIIA), one of the components of Salviamiltiorrhiza(Danshen), has anti-oxidative stress activity and is widely used to treat diabetes-associated diseases. However, its efficacy on DCM remains unclear. The present study aimed to investigate the potential therapeutic function of TSIIA on DCM in an experimental diabetic rat model. Streptozotocin (STZ)-induced diabetic rats were intraperitoneally injected with TSIIA for 6 weeks. The present results indicated that blood glucose concentration was slightly reduced in the low-dose TSIIA treatment group. TSIIA injection was also noted to improve cardiac function, and restore loss of mitochondrial cristae, swollen mitochondrial matrix and disorganized myofibrils in myocardial cells, which are thought to be characteristics of apoptosis. Furthermore, TSIIA injection could increase the activity of superoxide dismutase in STZ-induced diabetic rats, and suppress the endoplasmic reticulum (ER) stress signaling pathway via reducing the expression of glucose-regulated protein 78 and $\mathrm{C} / \mathrm{EBP}$ homologous protein. These results provide evidence that TSIIA may ameliorate DCM in diabetic rats, possibly via suppressing oxidative stress and ER stress activation.
\end{abstract}

\section{Introduction}

Diabetes mellitus (DM) is a chronic metabolic disease that has become a major health problem worldwide. In 2014, an estimated 387 million individuals worldwide were diagnosed with DM and the prevalence is expected to rise to 592 million

Correspondence to: Professor Yueming Zhang, Basic Medical College, Zhejiang Chinese Medical University, 548 Binwen Road, Hangzhou, Zhejiang 310053, P.R. China

E-mail: zyueming@vip.sina.com

Key words: diabetic cardiomyopathy, Tanshinone IIA, glucose-regulated protein $78, \mathrm{C} / \mathrm{EBP}$ homologous protein by 2035 (1). In addition, patients with DM have a high mortality rate that is often caused by diabetic cardiomyopathy (DCM) $(2,3)$.

DCM is characterized by left ventricular hypertrophy and reduced diastolic function, and is a major complication in patients with either type 1 or type 2 DM (4). Previous studies have demonstrated that diverse pathogenic mechanisms contribute to DCM, including hyperglycemia, inflammation, fibrosis and apoptosis $(5,6)$. Among these factors, cardiomyocyte apoptosis is thought to initiate cardiac remodeling and results in cardiac dysfunction (7). Therefore, it serves a key function in the pathogenesis and progression of DCM. Accumulating evidence has indicated that cardiomyocyte endoplasmic reticulum (ER) stress is involved in the pathogenesis of cardiac dysfunction in patients with DM and animal models $(8,9)$. The early stages of ER stress are considered to be an adaptive response aiming at maintenance of ER homeostasis. This is known as the unfolded protein response (UPR), which is monitored by glucose-regulated protein 78 (Grp78), an ER chaperone (10). If it persists long-term, ER stress could induce the intrinsic pathway of apoptosis (11). C/EBP homologous protein (CHOP), a marker of ER stress, was identified to be elevated in multiple organs of a diabetic animal model, including myocardial tissue $(9,12,13)$. Furthermore, CHOP knockout mice were observed to exhibit less pronounced hypertrophy and cardiac dysfunction in comparison with wild-type animals (14).

Tanshinone IIA (TSIIA), a phytochemical derived from the roots of Salvia miltiorrhiza, has the effect of suppressing apoptosis via multiple pathways $(15,16)$. In previous studies, it has been demonstrated to inhibit ER-induced apoptosis in certain tissues $(17,18)$. Therefore, it was hypothesized that TSIIA could improve cardiac function by inhibiting ER stress-induced apoptosis. The present study performed comparative studies on ER stress-associated signaling proteins Grp78 and $\mathrm{CHOP}$ to explore the mechanism of TSIIA on protecting cardiac function in a streptozotocin (STZ)-induced diabetic rat model.

\section{Materials and methods}

Animals. The experimental procedures were reviewed and approved by the Committee for the Care and Use of Laboratory 
Animals at Zhejiang Chinese Medical University (Hangzhou, China). A total of 40 6-week-old male Sprague-Dawley rats (160-180 g; Shanghai SLAC Laboratory Animal Co., Ltd., Shanghai, China) were individually housed in separately ventilated cages at a temperature of $23 \pm 2^{\circ} \mathrm{C}$ and humidity of $55 \pm 2 \%$ with a 12 -h light/dark cycle, and were given free access to standard food and water.

Materials. TSIIA was purchased from Chiatai Qingchunbao Pharmaceutical Co., Ltd., (Hangzhou, China). STZ was purchased from Sigma-Aldrich (Merck KGaA, Darmstadt, Germany). The blood glucose test machine (FreeStyle Optium $\mathrm{Neo}$ ) and strips were purchased from Abbott Laboratories (Lake Bluff, IL, USA). Grp78 (cat. no. 3813) and CHOP (cat. no. 2895) antibodies were purchased from Cell Signaling Technology, Inc., (Danvers, MA, USA). The $\beta$-actin antibody (cat. no. AbD12141) was purchased from Bio-Rad Laboratories, Inc., (Hercules, CA, USA).

Induction of diabetes in rats. A total of 40 male rats were randomized into the following groups ( $\mathrm{n}=10$ per group): Control group, DM group, DM rats treated with lower-dose TSIIA (L-TSIIA group) and DM rats treated with high-dose TSIIA (H-TSIIA group). DM was induced by a single intraperitoneal injection of STZ (dissolved in $10 \mathrm{mM}$ citrate buffer, $\mathrm{pH} 4.5$ ) at a dosage of $60 \mathrm{mg} / \mathrm{kg}$ body weight to induce diabetes. Rats in groups with TSIIA injection were intraperitoneally administered daily at a dose of $2 \mathrm{mg} / \mathrm{kg}$ (L-TSIIA group) and $4 \mathrm{mg} / \mathrm{kg}$ (H-TSIIA group) for 6 weeks, while the rats in the control and diabetes groups were injected with the same volume of vehicle (PBS). At day 2 after STZ injection, tail-vein blood glucose concentration was measured. Rats with blood glucose level $>16 \mathrm{mmol} / \mathrm{l}$ were identified as diabetic model rats. Body weight and fasting blood glucose were determined at the end of week 6 . TSIIA was dissolved in DMSO at a concentration of $10 \mathrm{mg} / \mathrm{ml}$ and then, diluted to $0.5 \mathrm{mg} / \mathrm{ml}$ with PBS prior to injection.

Blood and tissue sample preparation. At the end of week 6 following STZ injection, rats were anesthetized in an induction chamber with 2-3\% isoflurane (Abbott Laboratories), then intraperitoneally injected with $1 \%$ pentobarbital sodium salt (30 mg/kg; Sigma-Aldrich; Merck KGaA). Blood was collected from carotid arteries and left at room temperature for $1 \mathrm{~h}$. The blood samples were subsequently centrifuged at $1,500 \mathrm{x}$ g for $15 \mathrm{~min}$, followed by the separation of serum from the blood cells. Serum was preserved at $-20^{\circ} \mathrm{C}$ for evaluating high-sensitivity superoxide dismutase (SOD), which was performed using a SOD assay kit (cat. no. A001-1-1; NanJing JianCheng Bioengineering Institute, Nanjing, China) After collecting blood, all rats were perfused with $50 \mathrm{ml}$ saline. Next, 5 rats in each group were perfused with $200 \mathrm{ml} 4 \%$ paraformaldehyde solution for subsequent transmission electron microscopy (TEM) observation. The myocardial tissue from another 5 rats (in each group) was preserved in liquid nitrogen for detecting the expression of relevant genes and proteins by reverse transcription-quantitative polymerase chain reaction (RT-qPCR) and western blotting, respectively.

TEM. Following perfusion with $4 \%$ paraformaldehyde, the myocardial tissue from the left ventricle was dissected, then rinsed in $2.5 \%$ glutaraldehyde at $4{ }^{\circ} \mathrm{C}$ for $4 \mathrm{~h}$. The tissues were rinsed in buffer and post-fixed with $1 \%$ osmium tetroxide for $1 \mathrm{~h}$ at room temperature, dehydrated in graded alcohol, then transferred to propylene oxide. The tissues were embedded gradually in blocks of Epon 812 resin for 2 days at $60^{\circ} \mathrm{C}$ undergoing a graded ethanol dehydration series, and infiltrated using a mixture of $50 \%$ propylene oxide and $50 \%$ resin overnight. Then, $24 \mathrm{~h}$ later, the tissues were embedded in pure resin for 2 days at $60^{\circ} \mathrm{C}$. Then, $120 \mathrm{~nm}$-thick sections were cut using a histology diamond knife on an Ultracut E microtome (Leica Microsystems $\mathrm{GmbH}$, Wetzlar, Germany) and stained with $4 \%$ uranyl acetate for $20 \mathrm{~min}$ and with $0.5 \%$ lead citrate for $5 \mathrm{~min}$ at room temperature. The ultrastructure of mitochondria and myofibrils was observed under TEM (Philips Tecnai 10; Philips Medical Systems B.V., Eindhoven, The Netherlands). Mitochondrial subpopulation densities were determined within a defined region $\left(100 \mu \mathrm{m}^{2}\right)$ with a minimum of 12 images in each group, which were taken at x5,900 magnification, as described previously (19). Analysis was performed using Image $\mathbf{J}$ software (v.1.46; National Institutes of Health, Bethesda, MD, USA).

Western blotting. Total protein from rat myocardium tissues was extracted using ice-cold RIPA buffer (cat. no. P0013B; Beyotime Institute of Biotechnology, Haimen, China). Protein concentration was determined using a BCA Protein Assay Reagent kit (cat. no. 23225; Pierce; Thermo Fisher Scientific, Inc.). Protein $(30 \mu \mathrm{g})$ was separated by $12 \%$ SDS-PAGE and transferred to a PVDF membrane. The membranes were blocked with $5 \%$ non-fat milk for $2 \mathrm{~h}$ at room temperature, then incubated overnight at $4^{\circ} \mathrm{C}$ with primary antibodies (monoclonal anti-CHOP (cat. no. 2895; Cell Signaling Technology, Inc.; 1:1,000) and polyclonal GRP-78 (1:1,000; cat. no. 3177; Cell Signaling Technology, Inc.). After three washes with TBST, blots were incubated with horseradish peroxidase conjugated anti-mouse secondary antibodies $(1: 10,000$; cat. no. BA1050; Wuhan Boster Biological Technology, Ltd., Wuhan, China) and horseradish peroxidase conjugated anti-rabbit secondary antibody $(1,10,000$; cat. no. BA2006; BOSTER Biological Tech Co., Ltd., Wuhan, China) for $1 \mathrm{~h}$ at room temperature. The immunoreactive bands were visualized using a chemiluminescent reagent as recommended by the Supersignal West Dura Extended Duration Substrate kit (cat. no. 34075, Pierce; Thermo Fisher Scientific, Inc.). The signals of the bands were quantified using the VICTOR-Z 1420 multilabel counter (PerkinElmer, Inc., Waltham, MA, USA). Equal protein loading in each lane was confirmed by hybridization with a $1: 10,000$ dilution of $\beta$-actin antibody (cat. no. AbD12141; Bio-Rad Laboratories, Inc.). The results were expressed as density relative to $\beta$-actin as determined via Image J software (v.1.46).

$R T-q P C R$. Myocardial tissue preserved in liquid nitrogen was used for RT-qPCR analysis. Total RNA was isolated from the specimens using the TRIzol reagent kit (Invitrogen; Thermo Fisher Scientific, Inc.) according to the manufacturer's protocol. Total RNA $(1 \mu \mathrm{g})$ was reverse transcribed to cDNA using the Transcriptor First Strand cDNA Synthesis kit (Takara Biotechnology Co., Ltd., Tokyo, Japan). qPCR was performed using Power SYBR Green qPCR Master mix (Applied Biosystems; Thermo Fisher Scientific, Inc.). cDNA was 
Table I. Effect of TSIIA on body weight and blood glucose concentration.

\begin{tabular}{|c|c|c|c|c|c|c|}
\hline \multirow[b]{2}{*}{ Group } & \multicolumn{3}{|c|}{ Body weight (g) } & \multicolumn{3}{|c|}{ Blood glucose concentration (mmol/l) } \\
\hline & $\begin{array}{l}\text { Prior to STZ } \\
\text { injection }\end{array}$ & $\begin{array}{c}2 \text { days post-STZ } \\
\text { injection }\end{array}$ & $\begin{array}{c}6 \text { weeks post-STZ } \\
\text { injection }\end{array}$ & $\begin{array}{l}\text { Prior to STZ } \\
\text { injection }\end{array}$ & $\begin{array}{l}2 \text { days post-STZ } \\
\text { injection }\end{array}$ & $\begin{array}{c}6 \text { weeks post-STZ } \\
\text { injection }\end{array}$ \\
\hline Control & $133.27 \pm 3.67$ & $141.59 \pm 4.92$ & $404.85 \pm 31.48$ & $5.71 \pm 0.55$ & $6.25 \pm 0.64$ & $5.67 \pm 0.43$ \\
\hline Diabetes & $134.16 \pm 4.03$ & $142.02 \pm 5.63$ & $299.26 \pm 47.14^{\mathrm{a}}$ & $5.64 \pm 0.66$ & $21.87 \pm 3.35^{\mathrm{a}}$ & $25.07 \pm 2.59^{\mathrm{a}}$ \\
\hline L-TSIIA & $136.40 \pm 4.23$ & $144.20 \pm 5.51$ & $311.99 \pm 49.92^{\mathrm{a}}$ & $6.12 \pm 0.79$ & $21.78 \pm 4.73^{\mathrm{a}}$ & $20.78 \pm 2.88^{\mathrm{a}, \mathrm{b}}$ \\
\hline H-TSIIA & $130.08 \pm 5.39$ & $138.68 \pm 6.55$ & $289.50 \pm 42.18^{\mathrm{a}}$ & $5.68 \pm 0.52$ & $23.89 \pm 4.18^{\mathrm{a}}$ & $23.46 \pm 3.05^{\mathrm{a}}$ \\
\hline
\end{tabular}

Data are presented as the mean \pm standard deviation $(\mathrm{n}=10) .{ }^{\mathrm{a}} \mathrm{P}<0.01$ vs. control group; ${ }^{\mathrm{b}} \mathrm{P}<0.05$ vs. diabetes group. TSIIA, Tanshinone IIA; STZ, streptozotocin; Diabetes, STZ-induced diabetic rats; L-TSIIA, diabetic rat treated with low dose TSIIA (2 mg/kg/day); H-TSIIA, diabetic rat treated with high dose TSIIA (4 mg/kg/day).

denatured by heating samples to $94^{\circ} \mathrm{C}$ for 3 min. The template was amplified by 40 rounds of PCR (denaturation at $94^{\circ} \mathrm{C}$ for $10 \mathrm{sec}$, annealing at $60^{\circ} \mathrm{C}$ for $30 \mathrm{sec}$ and extension at $72^{\circ} \mathrm{C}$ for $30 \mathrm{sec}$ ) before measuring fluorescence at $72^{\circ} \mathrm{C}$. PCR primers (Invitrogen; Thermo Fisher Scientific, Inc.) for ER stress-associated genes: Grp-78 (forward, 5'-GGTGCAGCAGGACAT CAAGTT-3'; reverse, 5'-CCCACCTCCAATATCAACTTG A-3'), CHOP (forward, 5'-CTGCCTTTCACCTTGGAGAC-3'; reverse, 5'-CGTTTCCTGGGGATGAGATA-3') and GAPDH (forward 5'-GGTGGACCTCATGGCCTACAT-3' and reverse 5'-GCCTCTCTCTTGCTCTCAGTATCCT-3'). The comparative $\mathrm{C}_{\mathrm{q}}$ method was utilized as described previously (20).

Statistical analysis. Data are expressed as the mean \pm standard deviation. A two-tailed, unpaired Student's t-test was used to compare two groups. Multiple group comparisons were performed using one-way analysis of variance, followed by Newman-Keuls post-hoc test. $\mathrm{P}<0.05$ was considered to indicate a statistically significant difference. Data were analyzed using GraphPad Prism 6 software (GarphPad Software, Inc., La Jolla, CA, USA).

\section{Results}

Effect of TSIIA on body weight and blood glucose concentration. There were no significant differences among groups in terms of body weight or blood glucose concentration prior to STZ injection (Table I). However, body weight in diabetic rats was observed to be significantly decreased $(\mathrm{P}<0.01$; Table I) at 6 weeks when compared with the control. There were no significant differences between the L-TSIIA and H-TSIIA groups (Table I). Furthermore, blood glucose concentration was significantly increased at 2 days and 6 weeks following STZ injection in the diabetes group as compared with the control group $(\mathrm{P}<0.01$; Table I). TSIIA was beneficial for reducing blood glucose; the concentration of blood glucose was significantly decreased in the L-TSIIA group $(20.78 \pm 2.88 \mathrm{mmol} / \mathrm{l})$ at 6 weeks when compared with the diabetes group $(\mathrm{P}<0.05$; Table I).

TSIIA injection rescues injured mitochondrial structure and reduces mitochondrial density caused by hyperglycemia. TEM examination revealed that mitochondrial structure was
Table II. Quantification of mitochondrial density under transmission electron microscopy.

\begin{tabular}{lc}
\hline Groups & Mitochondrial density $/ 100 \mu \mathrm{m}^{2}$ \\
\hline Control & $47.56 \pm 12.90$ \\
Diabetes & $28.12 \pm 8.39^{\mathrm{a}}$ \\
L-TSIIA & $28.14 \pm 8.39^{\mathrm{a}}$ \\
H-TSIIA & $40.62 \pm 14.47^{\mathrm{b}}$ \\
\hline
\end{tabular}

Data are presented as the mean \pm standard deviation $(n=12) .{ }^{\mathrm{a}} \mathrm{P}<0.01$ vs. control group; ${ }^{\mathrm{b}} \mathrm{P}<0.05$ vs. diabetes group. TSIIA, Tanshinone IIA; Diabetes, streptozotocin-induced diabetic rats; L-TSIIA, diabetic rat treated with low dose TSIIA ( $2 \mathrm{mg} / \mathrm{kg} /$ day); H-TSIIA, diabetic rat treated with high dose TSIIA ( $4 \mathrm{mg} / \mathrm{kg} /$ day).

normal in the control group (Fig. 1A), while the mitochondrial cristae of the myocardial cells of the diabetic group disappeared and the vacuoles in the swollen mitochondrial matrix increased, which are thought to be characteristics of apoptosis (Fig. 1B). Furthermore, disordered myofibrils were observed in the myocardial cells of diabetic rats (Fig. 1B), but not the control group. Administration of TSIIA was observed to attenuate the damage induced by hyperglycemia (Fig. 1C and D). Furthermore, it was observed that the mitochondrial density was significantly decreased in the diabetes group $(\mathrm{P}<0.01)$, which was rescued by H-TSIIA treatment $(\mathrm{P}<0.05$ vs. diabetes group; Table II).

Expression of ER stress-associated proteins. The mRNA and protein expression levels of Grp78, an ER chaperone, and CHOP, a protein crucial to growth arrest and DNA damage, were measured. According to RT-qPCR and western blot analysis results, the gene and protein levels of Grp78 and CHOP were significantly enhanced in the diabetes group as compared with the control group $(\mathrm{P}<0.05$; Fig. 2). However, TSIIA application during treatment could reduce Grp78 and CHOP expression ( $\mathrm{P}<0.05$; Fig. 2). This suggested that ER stress was activated in myocardium tissue of diabetic rats, which could be suppressed by TSIIA treatment. These results indicate that TSIIA may be able to rescue injured 

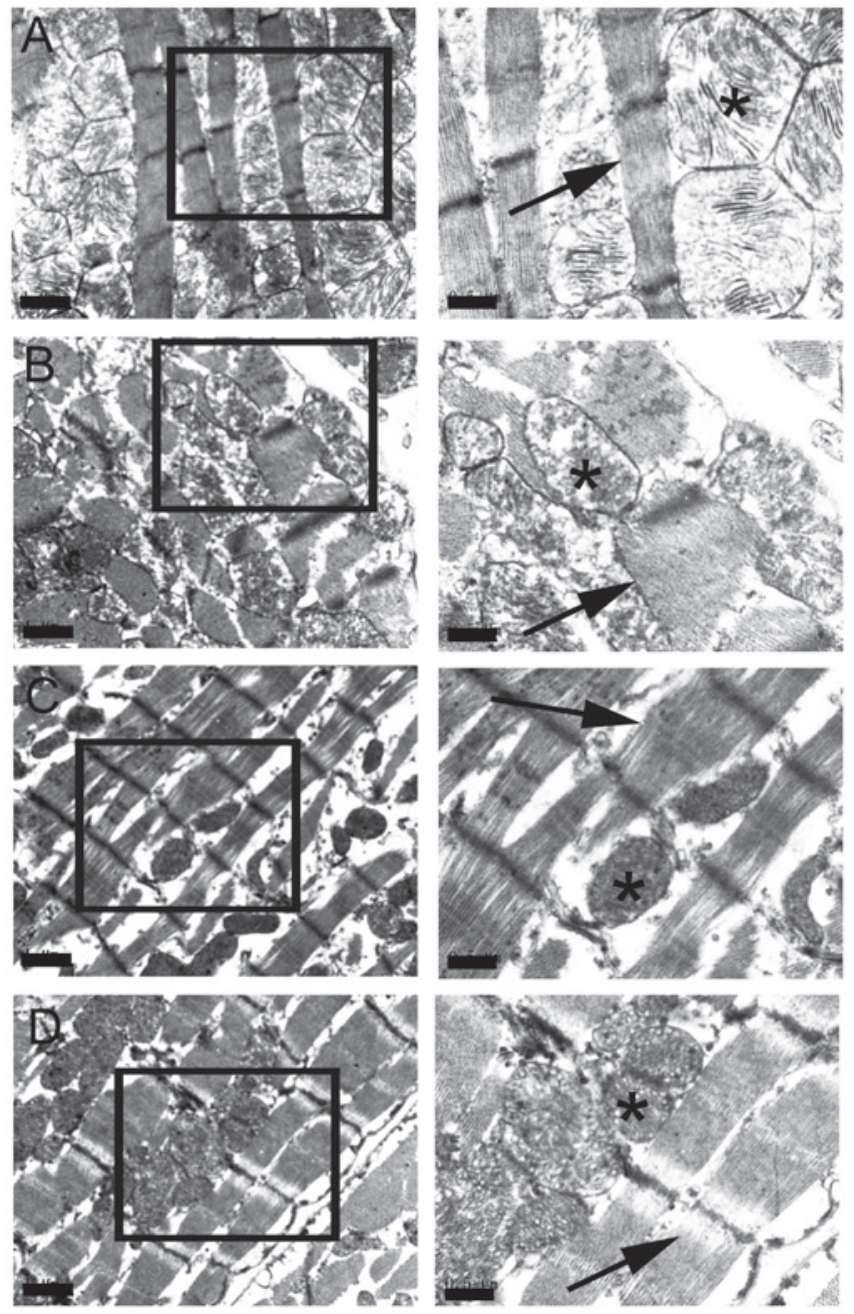

Figure 1. Transmission electron microscopy images depicting the morphology cardiomyocyte myofibrils and mitochondria. The inset in each picture is enlarged and displayed on the right. (A) Normal structure of mitochondrial and myofibrils were observed in the control group; mitochondrial cristae were clearly observed (indicated by the asterisk) and myofibrils were intact and in parallel rows (indicated by the arrow). (B) In the diabetes group, mitochondrial cristae of myocardial cells disappeared and there were vacuoles in the swollen mitochondrial matrix (indicated by asterisk). The myofibrils were disordered (indicated by arrow). TSIIA prevented the appearance of distorted myofibrils (indicated by the arrow) and swollen mitochondria (indicated by the asterisk) in the (C) L-TSIIA and (D) H-TSIIA groups. Scale bar: $1 \mu \mathrm{m}$ in left column; $0.5 \mu \mathrm{m}$ in right column. TSIIA, Tanshinone IIA; L-TSIIA, diabetic rat treated with lower-dose TSIIA ( $2 \mathrm{mg} / \mathrm{kg} /$ day); H-TSIIA, diabetic rat treated with high-dose TSIIA ( $4 \mathrm{mg} / \mathrm{kg} /$ day).

mitochondrial structure by hyperglycemia via inhibition of the ER stress response.

TSIIA reduces the expression of SOD. Oxidative stress could trigger ER stress activation (21). SOD, as an important antioxidant, was measured in the serum of rats to investigate the mechanism of TSIIA protective effects on DCM. As indicated in Fig. 3, SOD activity was significantly decreased in diabetic rats $(22.64 \pm 8.18 \mathrm{U} / \mathrm{ml})$ when compared with the control group $(60.74 \pm 16.92 \mathrm{U} / \mathrm{ml} ; \mathrm{P}<0.001)$. TSIIA treatment significantly increased SOD activity in L-TSIIA $(\mathrm{P}<0.001)$ and H-TSIIA rats $(\mathrm{P}<0.05)$ when compared with non-treated diabetic rats.

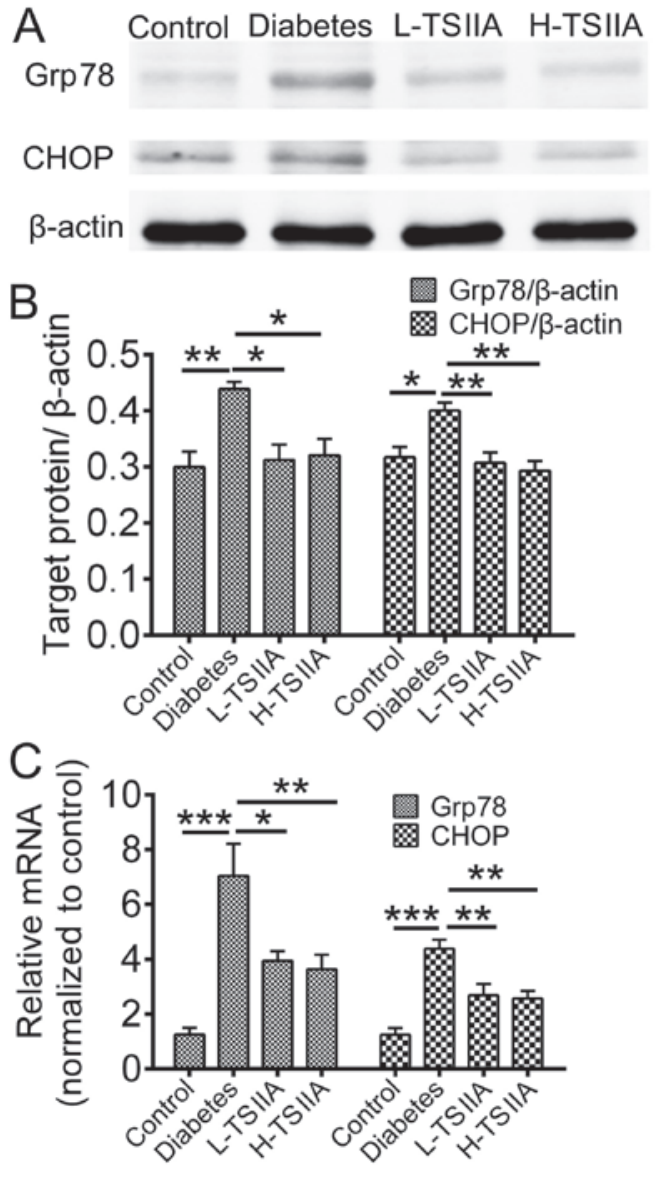

Figure 2. TSIIA suppresses the Grp78/CHOP pathway. (A) Western blot analysis of Grp78 and CHOP protein in myocardial tissue homogenates. Bands were normalized to $\beta$-actin. (B) Quantification of mean data from 5 rats. (C) mRNA expression of Grp78 and CHOP in myocardial tissue. " $\mathrm{P}<0.05$, ${ }^{* *} \mathrm{P}<0.01$ and ${ }^{* * * *} \mathrm{P}<0.001(\mathrm{n}=5)$. Grp78, glucose-regulated protein 78; CHOP, C/EBP homologous protein; TSIIA, Tanshinone IIA; L-TSIIA, diabetic rat treated with lower-dose TSIIA ( $2 \mathrm{mg} / \mathrm{kg}$ /day); H-TSIIA, diabetic rat treated with high-dose TSIIA ( $4 \mathrm{mg} / \mathrm{kg} /$ day).

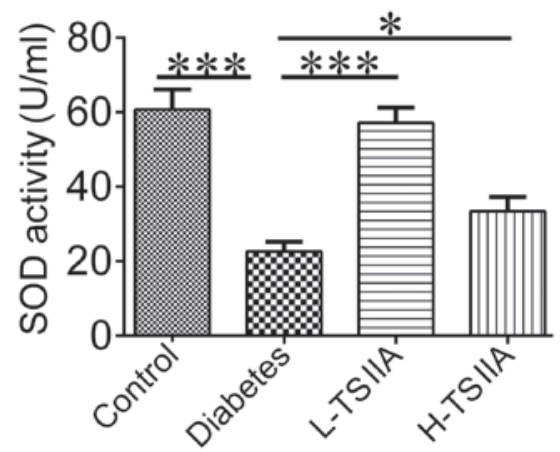

Figure 3. TSIIA increases the activity of SOD. SOD activity was measured in the serum of rats from each group. ${ }^{*} \mathrm{P}<0.05$ and ${ }^{* * * *} \mathrm{P}<0.001 \quad(\mathrm{n}=10)$. SOD, superoxide dismutase; TSIIA, Tanshinone IIA; L-TSIIA, diabetic rat treated with lower-dose TSIIA ( $2 \mathrm{mg} / \mathrm{kg} /$ day $)$; H-TSIIA, diabetic rat treated with high-dose TSIIA (4 mg/kg/day).

\section{Discussion}

DCM, which is now recognized to have a high prevalence among patients with diabetes, leads to structural and functional changes of myocardial tissue (22). The aim of the current 
study was to investigate the effect and mechanism of TSIIA on cardiac dysfunction in a diabetic rat model. The current results indicated that improved cardiac pathological changes were accompanied by rescued myocardial damage following TSIIA treatment. This suggested that TSIIA exerts a beneficial effect on DCM. The underlying mechanism was also investigated in the present study; to the best of our knowledge, this has not been reported previously.

As a key component of S. miltiorrhiza, TSIIA has been used to treat multiple diseases, including Alzheimer's disease, chronic kidney disease, cerebral ischemic injury and DCM (23-26). Furthermore, previous studies have demonstrated that TSIIA could attenuate myocardial ischemia reperfusion injury due to its antioxidant capacity (27). In the present study, it was observed that SOD activity was significantly reduced in the serum of diabetic rats compared with the control group, while TSIIA at different dosages could elevate the activity of SOD. SOD is known to be a major scavenger of superoxide radicals, which may attenuate oxidative stress (28). Therefore, it is proposed that TSIIA may alleviate the injury induced by hyperglycemia via increased SOD activity. In the current study, the ultrastructure of mitochondria and myofibrils was also observed under TEM. Previous studies have reported that STZ-induced hyperglycemia elevates free radicals and represses antioxidant defense mechanisms, which may lead to cell disruption, degenerated mitochondria and disordered myofibrils $(29,30)$ and may induce DCM after 6 weeks (31). Consistent with these findings, in the current study, a lack of mitochondrial cristae and disordered myofibrils were observed in diabetic rats, which was accompanied by a reduction in mitochondrial density. However, it was identified that mitochondrial cristae were partially restored, and mitochondrial density was rescued, by application of TSIIA, which may be associated with increased activity of SOD. A previous study also reported that mitochondrial structure plays an important role in cardiac function (32). Degradation in mitochondrial structure results in a progressive increase in mitochondrial $\mathrm{Ca}^{2+}$ sequestration and mitochondrial depolarization, finally leading to cardiac dysfunction (33).

Previous research has identified direct associations between hyperglycemia, oxidative stress and protein folding (21). Alterations in redox state could result in unfolded/misfolded proteins accumulating in the ER and UPR being initiated, generally referred as ER stress (34). Accumulation of unfolded/misfolded proteins could also increase reactive oxygen species production and therefore exacerbate ER stress. One major goal of UPR/ER stress is to induce ER chaperones that promote protein folding, which is controlled by the ER chaperone, Grp78 (35). Activation of Grp78 protein expression is a widely used indicator of ER stress. In the current study, the expression of Grp78 was upregulated in the myocardial tissue of diabetic rats, which was accompanied by the increased expression of CHOP, a key mediator of ER stress-induced apoptosis. These results suggested that ER stress was activated in myocardial tissue of the STZ-induced diabetic animal model. However, the expression of Grp78 was significantly decreased following TSIIA treatment when compared with that in the diabetic group. Furthermore, CHOP is involved in one of the ER stress-induced cell apoptosis signaling pathways that could trigger cell apoptosis (36). In the present study, TSIIA inhibited CHOP apoptosis signaling pathways. Therefore, it was suggested that TSIIA may alleviate cardiomyocyte ER stress-induced apoptosis.

The present results demonstrated that TSIIA may exert a therapeutic effect on DCM. TSIIA was observed to improve cardiac pathological changes, and this effect may be via inhibiting ER stress-mediated myocardial cell apoptosis.

\section{Acknowledgements}

Not applicable.

\section{Funding}

The present study was supported by the Natural Science Foundation of Zhejiang Province, China (grant no. LY 16H270003).

\section{Availability of data and materials}

All data generated or analyzed during the present study are included in this published article.

\section{Authors' contributions}

YZ designed the study. Diabetes animal model induction, blood and tissue sample preparation was performed by ST and LC. Sample preparation for TEM was done by JS, NZ and XS performed Western blot and real time PCR. RS and GG analyzed data. The manuscript was written by ST. All authors have read and approved the final manuscript.

\section{Ethics approval and consent to participate}

The experimental procedures were reviewed and approved by the Committee for the Care and Use of Laboratory Animals at Zhejiang Chinese Medical University (Hangzhou, China).

\section{Patient consent for publication}

Not applicable.

\section{Competing interests}

The authors declare that they have no competing interests.

\section{References}

1. Deng F, Wang S and Zhang L: Endothelial microparticles act as novel diagnostic and therapeutic biomarkers of diabetes and its complications: A literature review. Biomed Res Int 2016: 9802026, 2016

2. Boudina S and Abel ED: Diabetic cardiomyopathy, causes and effects. Rev Endocr Metab Disord 11: 31-39, 2010.

3. Li WF, Wang P, Li H, Li TY, Feng M and Chen SF: Oleanolic acid protects against diabetic cardiomyopathy via modulation of the nuclear factor erythroid 2 and insulin signaling pathways. Exp Ther Med 14: 848-854, 2017.

4. Asghar O, Al-Sunni A, Khavandi K, Khavandi A, Withers S, Greenstein A, Heagerty AM and Malik RA: Diabetic cardiomyopathy. Clin Sci (Lond) 116: 741-760, 2009. 
5. Westermann D, Walther T, Savvatis K, Escher F, Sobirey M, Riad A, Bader M, Schultheiss HP and Tschöpe C: Gene deletion of the kinin receptor B1 attenuates cardiac inflammation and fibrosis during the development of experimental diabetic cardiomyopathy. Diabetes 58: 1373-1381, 2009.

6. Falcao-Pires I and Leite-Moreira AF: Diabetic cardiomyopathy: Understanding the molecular and cellular basis to progress in diagnosis and treatment. Heart Fail Rev 17: 325-344, 2012.

7. Xia Y, Gong L, Liu H, Luo B, Li B, Li R, Li B, Lv M, Pan J and An F: Inhibition of prolyl hydroxylase 3 ameliorates cardiac dysfunction in diabetic cardiomyopathy. Mol Cell Endocrinol 403: 21-29, 2015.

8. Yang L, Zhao D, Ren J and Yang J: Endoplasmic reticulum stress and protein quality control in diabetic cardiomyopathy. Biochim Biophys Acta 1852: 209-218, 2015.

9. Zhang X, Liu S, Zhang G, Zhong M, Liu T, Wei M, Wu D, Huang X, Cheng Y, Wu Q and Hu S: Bariatric surgery ameliorates diabetic cardiac dysfunction by inhibiting ER stress in a diabetic rat model. Obes Surg 27: 1324-1334, 2017.

10. Xu LH, Xie H, Shi ZH, Du LD, Wing YK, Li AM, Ke Y and Yung WH: Critical role of endoplasmic reticulum stress in chronic intermittent hypoxia-induced deficits in synaptic plasticity and long-term memory. Antioxid Redox Signal 23: 695-710, 2015.

11. Iurlaro R and Muñoz-Pinedo C: Cell death induced by endoplasmic reticulum stress. FEBS J 283: 2640-2652, 2016.

12. Zhang X, Xu L, He D and Ling S: Endoplasmic reticulum stress-mediated hippocampal neuron apoptosis involved in diabetic cognitive impairment. Biomed Res Int 2013: 924327, 2013.

13. Liu X, Xu Q, Wang X, Zhao Z, Zhang L, Zhong L, Li L, Kang W, Zhang Y and Ge Z: Irbesartan ameliorates diabetic cardiomyopathy by regulating protein kinase D and ER stress activation in a type 2 diabetes rat model. Pharmacol Res 93: 43-51, 2015.

14. Minamino T, Komuro I and Kitakaze M: Endoplasmic reticulum stress as a therapeutic target in cardiovascular disease. Circ Res 107: 1071-1082, 2010.

15. Cui ZT, Liu JP and Wei WL: The effects of tanshinone IIA on hypoxia/reoxygenation-induced myocardial microvascular endothelial cell apoptosis in rats via the JAK2/STAT3 signaling pathway. Biomed Pharmacother 83: 1116-1126, 2016.

16. Zhang Z, Li Y, Sheng C, Yang C, Chen L and Sun J: Tanshinone IIA inhibits apoptosis in the myocardium by inducing microRNA-152-3p expression and thereby downregulating PTEN. Am J Transl Res 8: 3124-3132, 2016.

17. Zhang X, He D, Xu L and Ling S: Protective effect of tanshinone IIA on rat kidneys during hypothermic preservation. Mol Med Rep 5: 405-409, 2012

18. Yang GL, Jia LQ, Wu J, Ma YX, Cao HM, Song N and Zhang N: Effect of tanshinone IIA on oxidative stress and apoptosis in a rat model of fatty liver. Exp Ther Med 14: 4639-4646, 2017.

19. Ji W, Chen X, Lv J, Wang M, Ren S, Yuan B, Wang B and Chen L: Liraglutide exerts antidiabetic effect via PTP1B and $\mathrm{PI} 3 \mathrm{~K} / \mathrm{Akt} 2$ signaling pathway in skeletal muscle of KKAy Mice. Int J Endocrinol 2014: 312452, 2014

20. Livak KJ and Schmittgen TD: Analysis of relative gene expression data using real-time quantitative PCR and the 2(-Delta Delta C(T)) method. Methods 25: 402-408, 2001.

21. Nakka VP, Prakash-Babu P and Vemuganti R: Crosstalk between endoplasmic reticulum stress, oxidative stress, and autophagy: Potential Therapeutic targets for acute CNS injuries. Mol Neurobiol 53: 532-544, 2016.
22. Goyal BR and Mehta AA: Diabetic cardiomyopathy: Pathophysiological mechanisms and cardiac dysfuntion. Hum Exp Toxicol 32: 571-590, 2013.

23. Wang ZY, Liu JG, Li H and Yang HM: Pharmacological effects of active components of chinese herbal medicine in the treatment of Alzheimer's disease: A review. Am J Chin Med 44: 1525-1541, 2016.

24. Jiang C, Zhu W, Yan X, Shao Q, Xu B, Zhang M and Gong R: Rescue therapy with Tanshinone IIA hinders transition of acute kidney injury to chronic kidney disease via targeting GSK3 $\beta$. Sci Rep 6: 36698, 2016.

25. Wen PY, Li J, Lu BL, Liu J, Yang FZ, Zhou L, Luo H, Li WW and Zhou J: Tanshinone IIA increases levels of NeuN, protein disulfide isomerase, and $\mathrm{Na}+\mathrm{K}+-\mathrm{ATPase}$ and decreases evidence of microglial activation after cerebral ischemic injury. Neuroreport 27: 435-444, 2016.

26. Sun D, Shen M, Li J, Li W, Zhang Y, Zhao L, Zhang Z, Yuan Y, Wang $\mathrm{H}$ and Cao F: Cardioprotective effects of tanshinone IIA pretreatment via kinin B2 receptor-Akt-GSK-3 $\beta$ dependent pathway in experimental diabetic cardiomyopathy. Cardiovasc Diabeto 10: 4, 2011.

27. Li Q, Shen L, Wang Z, Jiang HP and Liu LX: Tanshinone IIA protects against myocardial ischemia reperfusion injury by activating the PI3K/Akt/mTOR signaling pathway. Biomed Pharmacother 84: 106-114, 2016.

28. Slemmer JE, Shacka JJ, Sweeney MI and Weber JT: Antioxidants and free radical scavengers for the treatment of stroke, traumatic brain injury and aging. Curr Med Chem 15: 404-414, 2008

29. Kara A, Unal D, Simsek N, Yucel A, Yucel N and Selli J: Ultra-structural changes and apoptotic activity in cerebellum of post-menopausal-diabetic rats: A histochemical and ultra-structural study. Gynecol Endocrinol 30: 226-231, 2014.

30. Yang M, Xu Z, Zhang R, Zhang P, Weng Y, Shen Y and Zhang X: Protection of myocardium in streptozotocin-induced diabetic rats by water extracts of Hsian-tsao (Mesona procumbens Hemsl.). Asia Pac J Clin Nutr 17: 23-29, 2008.

31. Liu XY, Liu FC, Deng CY, Zhang MZ, Yang M, Xiao DZ, Lin QX, Cai ST, Kuang SJ, Chen J, et al: Left ventricular deformation associated with cardiomyocyte $\mathrm{Ca}(2+)$ transients delay in early stage of low-dose of STZ and high-fat diet induced type 2 diabetic rats. BMC Cardiovasc Disord 16: 41, 2016.

32. Kyrychenko V, Poláková E, Janíček R and Shirokova N: Mitochondrial dysfunctions during progression of dystrophic cardiomyopathy. Cell Calcium 58: 186-195, 2015.

33. Wang HP, Zhang WH, Wang XF, Zhu J, Zheng YQ, Xia Q and Zhi JM: Exposure to AT1 receptor autoantibodies during pregnancy increases susceptibility of the maternal heart to postpartum ischemia-reperfusion injury in rats. Int J Mol Sci 15: 11495-11509, 2014

34. Pluquet O, Pourtier A and Abbadie C: The unfolded protein response and cellular senescence. A review in the theme: Cellular mechanisms of endoplasmic reticulum stress signaling in health and disease. Am J Physiol Cell Physiol 308 C415-C425, 2015.

35. Ron D and Walter P: Signal integration in the endoplasmic reticulum unfolded protein response. Nat Rev Mol Cell Biol 8: 519-529, 2007.

36. Sano R and Reed JC: ER stress-induced cell death mechanisms. Biochim Biophys Acta 1833: 3460-3470, 2013. 